\title{
LA FORME DE RESISTANCE DES TRICHOMONAS HUMAINS
}

\author{
Par R. MANDOUL, R. PAUTRIZEL et R. DARGELOS
}

Bien que parasites banaux de la bouche, de l'intestin et du vagin, les Trichomonas humains, pourtant facilement cultivables, n'ont pas encore livré entièrement le secret de leur cycle biologique. Leurs kystes demeurent, en effet, inconnus, et ceci malgré l'assertion de quelques auteurs tels que Bensen, Schmidt et Kamniker. Aussi bien, le mode de contamination de l'homme demeure-t-il assez mystérieux, si l'on se souvient de la fragilité des trophozoïdes qui meurent rapidement dans le milieu extérieur. On a cependant depuis longtemps remarqué que lorsque les conditions de vie deviennent défavorables, ces micro-organismes subissent des modifications morphologiques particulières. Nous nous proposons d'étudier quelles sont ces modifications et quelle peut être leur signification biologique.

En 1915, K. M. Lynch signale que, maintenus pendant quelques heures à la température ambiante, ces Trichomonas de la bouche et du vagin s'arrondissent, tandis que les flagelles disparaissent. De son côté, C. M. Wenyon (1926), écrit dans sa Protozoology : 'si les flagellés ne dégénèrent pas, ils s'arrondissent progressivement et s'immobilisent ; ce sont ces Trichomonas arrondis que l'on suppose conduire à l'enkystement, mais on n'a pas vu de stade enkysté chez le Trichomonas intestinal. » H. C. Hinshaw (1927), travaillant sur le parasite de la bouche, note simplement l'immobilisation du protiste de culture à la température de $0^{\circ}$, ainsi que son gonflement dans l'eau pure, tandis qu'il devient complètement sphérique. En 1932, P. B. Bland et ses collaborateurs considèrent les pseudo-kystes observés chez Trichomonas vaginalis, comme des formes de dégénérescence du flagellé. Si l'on abandonne ces formes pendant quelques jours, disent-ils, elles disparaissent du milieu, ce qui prouve qu'elles ont été décomposées ; de plus, elles ne sont pas cultivables, et les auteurs concluent : "Bien que ces pseudo-kystes ne persistent pas, ils représentent peut-être une tentative vers la forme kystique que leurs ancêtres arrivaient sans doute à donner. $\gg$ Enfin, C. Dobell (1934), indique que les cultures du Trichomonas intestinal, après

Ann, de Parasitolocie, T. XXI, Nos 5-6, 1946, p. 241-245. 
séjour à la chambre froide à $10^{\circ}$, ne contiennent plus que des formes arrondies, immobiles, dépourvues de flagellés et de membrane ondulante. Il précise que si un grand nombre de ces éléments sont morts, il en est toutefois encore de vivants, car par repiquage sur milieu neuf à $37^{\circ}$, une riche culture peut toujours ètre obtenue.

Utilisant comme Hinshaw des cultures de Trichomonas de la bouche, nous avons fait agir sur elles divers agents susceptibles de contrarier leur vie végétative, dans le but d'obtenir des modifications morphologiques précédemment observées et d'en étudier la résistance.

Les cultures de 8 jours, à leur sortie de l'étuve à $37^{\circ}$, renferment un grand nombre de flagellés très mobiles, légèrement ovoïdes, gorgés de grains d'amidon. Leurs dimensions moyennes oscillent entre 7 et $15 \mu$. Les flagelles sont souvent apparents, les ondulations de la membrane ondulante sont plus rarement discernables; l'axostyle émerge à l'opposé du pôle flagellaire. La structure interne, difficile à détailler dans ces conditions, révèle parfois le noyau, mais le plus souvent celui-ci est dissimulé par les grains d'amidon ; le cytoplasme est homogène et granuleux.

Action du froid. - Si on porte de telles cultures à $4^{\circ}$, on note, au bout de vingt-quatre heures, que le nombre des flagellés a beaucoup diminué. Certains ont conservé leur forme habituelle, par contre, la plupart sont nettement sphériques ; chez ces derniers, les flagelles, peu actifs, n'impriment plus au micro-organisme que des mouvements très lents. La saillie de l'axostyle a disparu. Après trois jours, il' n'y a plus de formes mobiles, seuls subsistent des éléments sphérique, inertes, de 12 à $15 \mu$ de diamètre, à membrane cytoplasmique bien nette. En frappant de petits coups sur la lame on peut faire basculer ces éléments ; ils roulent alors dans le liquide ambiant en montrant une surface lisse, hoirs de laquelle aucun organite n'émerge. Dans l'intérieur de la sphère, à l'homogénéité initiale du eytoplasme, a succédé une hétérogénéité marquée : un ectoplasme hyalin, d'épaisseur variable, borde la face interne de la membrane protoplasmique, emprisonnant une portion centrale moins claire, finement granuleuse et renfermant en général, avec le noyau subcentral, plusieurs grains d'amidon. Parfois le noyau présente un grand nombre de ponctuations réfringentes.

Action de l'eau et d'une solution saline hypertonique. - Immergés dans de l'eau ordinaire ou distillée, les flagellés de culture s'arrondissent bientôt en augmentant de volume. Il y a done absorption d'eau par osmose. Leur taille peut alors atteindre $22 \mu$. La motilité, bien 
que fortement amoindrie, persiste pendant un certain temps. Au bout de 18 heures, le nombre des parasites a bien diminué. Ceux qui subsistent sont sphériques, immobiles, dépourvus de tout appendice extérieur. Ils sont enfin détruits, les uns après les autres, par rupiture de la membrane cellulaire et se vident de leur contenu. Après 24 heures, ils ont tous pratiquement disparu. En milieu hypertonique, l'éclatement du flagellé se réalise de même rapidement, pour la plupart des individus. Ceux qui peuvent résister prennent, en quelques minutes, la forme ronde sans modifier leurs dimensions, ce qui prouve que la membrane s'oppose, pendant un certain temps, à l'appauvrissement en eau du cytoplasme.

Action d'une solution chlorhydrique. - La transformation progressive des trophozoïdes très mobiles en formes sphériques qui tendent à s'immobiliser, peut être suivie à l'étude de Foot, lorsqu'on met les protistes au contact d'une solution à 2 p. 1.000 d'acide chlorhydrique dans du liquide de Ringer. Ce fait est intéressant à mentionner, car on sait que l'acidité gastrique normale est de $1 \mathrm{gr} .6$ à $1 \mathrm{gr} .7$ d'acide chlorhydrique p. 1.000. Dans une solution plus concentrée (4 p. 1.000), tout mouvement cesse en 10 minutes en même temps que les flagellés disparaissent ; les formes sphériques et inertes qui se conștituent subsistent pendant 45 minutes environ.

Action des matières fécales. - Le Trichomonas de la bouche, en présence d'une dilution fécale à la température ambiante, se maintient pendant 30 heures. Les formes sphériques immobiles se manifestent au bout de quelques heures et se substituent peu à peu aux formes végétatives mobiles qui disparaissent complètement.

Nous constatons done que le trophozoïte, lorsqu'il subit des conditions de vie défavorab̧les, affecte une forme sphérique immobile. Il $\mathrm{y}$ a, en mème temps, disparition des flagelles, de la membrane ondulante, de l'axostyle, e'est-à-dire un processus de simplification analogue à celui qui s'accomplit au cours de l'enkystement des protozcaires intestinaux. Mais le noyau reste toujours unique. Il ne semble pas qu'il y ait mise en réserve de glycogène. Par contre, on note une assimilation progressive des grains d'amidon ingẻrés. Ces modifications morphologiques et ce comportement ont un caractère général et constant chez tous les Trichomonas humains. Quelle signification biologique peut-on attribuer à ces formes ? Sommes-nous en présence d'images léthales, d'aspects de dégénérescence ? Certainement pas, tout au moins pour quelques-uns de ces éléments. En effet, l'épreuve de Kuenen et Swellengrebel montre que certains d'entre eux sont parfaitement imperméables à une solution d'éosine à $2 \mathrm{p}$. 100 , tandis que beaucoup d'autres se colorent. 
Ce test de vitalité est confirmé d'une façon absolue par la possibilité de repiquage. A partir de ces formes sphériques, à l'exclusion de toute forme végétative, nous avons obtenu en effet, régulièrement, des cultures florissantes : jusqu'au dixième jour dans les cultures soumises au froid, jusqu'à la vingt-quatrième heure dans l'émulsion fécale, jusqu'à la soixantième minute dans la solution chlorhydrique à 2 p. 1.000 ; après contact avec l'eau distillée, Hinshaw, de son còté, a repiqué avec succès au bout de 12 heures. Ces divers chiffres témoignent d'une capacité de résistance bien marquée du flagellé sous sa forme sphérique, immobile, alors que le trophozoïte a été depuis longtemps éliminé. II faut cependant insister sur ce fait qu'un petit nombre seulement de ces éléments conserve vitalité et résistance, tandis que la plupart d'entre eux ne sont plus que des formes !éthales. L'explication que l'on peut donner à cette divergence est, selon nous, la suivante : dans les conditions expérimentales où nous sommes placés, nous exerçons une action brutale sur des formes végétatives qui se trouvent à divers stades de leur développement. Elles réagissent toutes de la même façon en s'arrondissant et en s'immobilisant, mais seuls les parasites parvenus à un cortain degré de maturité peuvent donner des formes sphériques viables. Les autres dégénèrent rapidement parce qu'ils ne sont pas encore physiologiquement aptes à mener à bien cette transformation. Quoi qu'il en soit, certains de ces éléments sphériques inertes constituent bien la forme de résistance des Trichomonas humains et peuvent être considérés comme les agents de dissémination assurant normalement la pérennité de l'espèce.

Peut=on leur attribuer la valeur de kystes ? Nous ne le pensons pas, car ils restent évidemment plus fragiles que les kystes des autres protozoaires intestinaux. Leur membrane cytoplasmique n'acquiert jamais cette solidité des membranes kystiques qui leur confère le rôle de coque protectrice. La dessiccation, les manœuvres d'enrichissement des selles par le Telemann-Rivas et aussi les mélanges fixateurs les altèrent rapidement, ce qui explique les échecs des tentatives de coloration. On peut les considérer seulement comme des ébauches de kystes.

En terminant, nous ne saurions mieux faire que d'adopter, vis-àvis du Trichomonas de la bouche, les conclusions déjà formulées par Dobell pour le parasite de l'intestin. Le Trichomonas de la bouche ne forme san's doute pas de kyste. Cependant les formes sphériques, aflagellées et gorgées d'amidon, offrent une résistance certaine à l'action des agents physiques et chimiques et sont physiologiquement équivalentes à des kystes. 


\section{RÉsumé}

Lorsqu'ils sont soumis expérimentalement à des conditions de vie défavorables, notamment à la réfrigération, les Trichomonas de la bouche, comme ceux du vagin et de l'intestin, donnent des formes sphériques, aflagellées, immobiles. Il ne s'agit pas de micro-organismes en dégénérescence, mais bien de formes ayant conservé leur vitalité, car elles sont parfaitement et pendant longtemps repiquables.

Ces formes sphériques n'ont cependant pas une résistance aussi considérable que celle des kystes et ne sauraient être considérées comme telles, mais, physiologiquement, elles semblent jouer le même rôle. Nous croyons qu'elles sont capables de remplacer les kystes qui paraissent faire défaut et d'assurer, en tant qu'agent d’hétéro-infestation, la pérennité de ces protozoaires.

\section{BIBLIOGRAPHIE}

Bl.ANd (P. B.), Golpstein (L.), WeNrich (D. H.) et Whiner (E.). - Am. J. Hyg., XVI, 1932 , p. $492-512$.

Dobel. (C.). - Parasitology, XXVI, 1934, p. 531-577.

Hinshaw (H. C.). - Univ. of Galif. Publ. in Zool., XXIX, 1926, p. 159-174, et XXXI, 1927, p. 31-51.

Lxхch (K. M.). - Am. J. trop. dis. and prevent. med., 1915, p. 62i-634.

WeNyox (C. M.). - Prolozoolog! , Londres, 1926 ; ef. I, p. 647-667.

Laboratoire de Parasitologie de la Faculté de Médecine de Bordeaux 